\title{
Multiconstraint Fuzzy Prediction Analysis Improved the Algorithm in Internet of Things
}

\author{
Xiaoya Deng $(\mathbb{D}$ \\ School of Intelligent Manufacturing, Sichuan University of Arts and Science, Da Zhou 635000, China \\ Correspondence should be addressed to Xiaoya Deng; dengxy@sasu.edu.cn
}

Received 12 April 2021; Revised 28 May 2021; Accepted 1 July 2021; Published 15 July 2021

Academic Editor: Chien-Ming Chen

Copyright (C) 2021 Xiaoya Deng. This is an open access article distributed under the Creative Commons Attribution License, which permits unrestricted use, distribution, and reproduction in any medium, provided the original work is properly cited.

\begin{abstract}
Multiconstraint prediction is a research hotspot and difficulty in the Internet of Things. Aiming at the main problems existing in the process of multiconstraint fuzzy prediction, this paper studies the related key technologies and methods and proposes an improved multiconstraint fuzzy prediction analysis algorithm in the application of Internet of Things. This paper introduces the multiconstraint attribute analysis, multiconstraint attribute normalization processing, multiconstraint attribute weight processing, multiconstraint attribute prediction analysis granularity setting, etc. and systematically calculates the ambiguity of multiconstraint attributes and realizes the multiconstraint fuzzy prediction analysis algorithm. Finally, combined with relevant cases, the algorithm is compared with the existing research results, which shows the effectiveness and feasibility of the algorithm in the Internet of Things.
\end{abstract}

\section{Introduction}

The Internet of Things (IOT) refers to the real-time collection of any object or process that needs monitoring, connection and interaction, and the collection of various required information such as sound, light, heat, electricity, mechanics, chemistry, biology, and location through various devices and technologies such as information sensors, radio frequency identification technology, global positioning system, infrared sensors, and laser scanners. Through all kinds of possible network access, the ubiquitous connection between things and people can be realized and the intelligent perception, identification and management of goods and processes can be realized. The Internet of Things is an information carrier based on the Internet and traditional telecommunication network. It enables all ordinary physical objects that can be independently addressed to form an interconnected network [1].

The Internet of Things (IOT) is the "Internet connected with everything." It is an extension and expansion network based on the Internet. It combines various information sensing devices with the network to form a huge network, realizing the interconnection of people, machines, and things at any time and any place [2].
The Internet of Things is an important part of the new generation of information technology. The IT industry is also called pan Internet, which means that things are connected and everything is connected. Thus, "the Internet of Things is the Internet of Things." This has two meanings: first, the core and foundation of the Internet of Things is still the Internet, which is an extended network based on the Internet, and second, its client extends to any item to exchange information and communicate with each other. Therefore, the definition of the Internet of Things is a kind of network that connects any article with the Internet according to the agreed protocol through information sensing devices such as RFID, infrared sensors, global positioning system, and laser scanner for information exchange and communication, so as to realize the intelligent identification, positioning, tracking, monitoring, and management of articles [3].

Constrained optimization, namely, constrained optimization problem, is a branch of optimization problem. It is to find a group of parameter values under a series of constraints, so that the target value of a function or a group of functions can reach the optimal value. The constraints can be either equality constraints or inequality constraints. The key to find this group of parameter values is to meet the constraints and 
target values to achieve the optimal value. The methods for solving constrained problems can be divided into traditional methods and evolutionary algorithms.

The basic idea of traditional methods, such as the Newton method and gradient method, is to transform a dynamic object to a static object and multiobjective into a single-object one, from point and face search. The traditional methods have the following problems: (1) the traditional gradientbased optimization methods (such as the feasible direction method and constrained variable scale method) usually seek a feasible and descending direction first, then search the linear in this direction, and repeat the steps above to get the optimal solution of the problem. However, the optimal solution is often local optimal. (2) For many practical constrained optimization problems, on the one hand, because the objective function is often complex, it is not only the problem with high dimension but also the existence of several small points in the optimization surface, which makes the traditional gradient-based algorithm difficult to work. On the other hand, the objective function is often discontinuous or differentiable in practical problems. Some problems have no analytic expression, so traditional algorithms find it difficult to solve these problems. And (3) because of the existence of constraints, the feasible search space of decision variables is irregular (such as nonconvex and unconnected), which increases the difficulty of searching the optimal solution, and sometimes, it is difficult to find the feasible solution.

The evolutionary algorithm is an intelligent global optimization method, which requires very low properties of the function itself, often only requires that the objective function value can be calculated, and does not require it to have continuity, differentiability, and other analytical properties. At the same time, it is based on the population evolution algorithm, so it can use the evolutionary algorithm to solve constrained optimization problems. The key of using the evolutionary algorithm to solve the constrained optimization problem is how to deal with constraints effectively, that is, how to balance the search between feasible and infeasible regions.

Common evolutionary algorithms for solving constrained optimization problems include the penalty function method, genetic algorithm, evolutionary strategy, evolutionary programming, and ant colony algorithm and particle swarm optimization.

In general, whether the evolutionary algorithm can converge to the global optimal solution is independent of the initial population, while the traditional optimization method depends on the initial solution. The evolutionary algorithm has the ability of global search, but many traditional optimization methods often fall into local optimum. The evolutionary algorithm has a wide range of applications and can effectively solve different types of problems, while traditional optimization methods can only solve a certain type of problems.

The shortcomings are as follows: (1) the parameters of the evolutionary algorithm, such as population size, evolutionary algebra, recombination probability, and mutation probability, often need to be set according to experience and are related to the problem to a certain extent and (2) the convergence problem of the evolutionary algorithm, the convergence judgment of the evolutionary algorithm in solving practical problems, is the lack of theoretical guidance.

Multiconstraint problem is a difficult problem in the process of system engineering analysis. In many engineering fields, it is necessary to optimize the design and analysis of multiconstraint problems, especially complex system engineering $[1,2]$. What is more complicated is that the development of complex systems is largely unknown due to the existence of multiple constraints. Therefore, it is very difficult to predict the development of complex systems scientifically, accurately, and reliably $[3,4]$, especially when the constraint information is fuzzy and uncertain. How to accurately capture information and make effective prediction, so as to provide good support for the design, maintenance, and repair service of the complex system, will have very important engineering application significance. At present, the research of fuzzy system prediction has analyzed some existing research results, especially literature $[5,6]$, based on the Grey Theory and neural network [7-9], vector machine, extension theory of genetic algorithm [10-12], etc., which has good engineering application effect.

Due to the uncertainty of fuzzy information, how to measure fuzzy information effectively and make more detailed and accurate information prediction still need further research. Therefore, based on the fuzzy system theory $[13,14]$, this paper analyzes the multiconstraint fuzzy prediction problem and proposes an improved multiconstraint fuzzy prediction analysis algorithm and model [15-19].

\section{The Basic Theory}

2.1. Multiconstraint Fuzzy Prediction Analysis Attribute Set. The content of multiple constraints has many meanings. First of all, in the process of design, analysis, and optimization of the complex system, the design objective is restricted by many factors. In order to get the ideal design result, we must deal with the relationship between design constraints and design objectives. Second, for complex systems, there are contradictions or incompatibilities among various types of design behaviors, design requirements, or design objectives. To deal with these contradictions or incompatibility, we must deal with the relationship between the factors involved. The third is the correlation between design attributes of complex systems. This association can be implicit or explicit, but it has an impact on the performance of complex systems. Therefore, in order to deal with the design problems better, we can extract the features and attributes of multiconstraints and form a multiconstraint fuzzy prediction analysis set, so that it can better predict and analyze the design objectives. Assuming that the extracted multiconstraint attributes are marked with the number $Q$, then, the multiconstraint fuzzy prediction analysis set $\mathbf{S}$ is expressed as follows:

$$
\mathbf{S}=\left\{S_{1}, S_{2}, \cdots, S_{k}, \cdots, S_{Q-1}, S_{Q}\right\} .
$$

2.2. The Fuzzy Distance. In order to describe the proximity between the predicted analysis target and the fuzzy set $\mathbf{S}$, the fuzzy system theory gives the concept of the fuzzy distance. Suppose that the fuzzy value of the constraint attribute $k$ of the prediction analysis target corresponding to the fuzzy 
set $\mathbf{S}$ is denoted as $v_{k}=\left[v_{k}(a), v_{k}(b)\right]$. The fuzzy interval of the fuzzy set $\mathbf{S}$ about the constraint attribute is denoted as $v_{k}^{S}=\left[v_{k}^{S}(a), v_{k}^{S}(b)\right]$. Then, the fuzzy distance between the two is denoted as

$$
D_{k}=\sqrt[P]{\frac{\left(\left|v_{k}^{S}(a)-v_{k}(a)\right|^{P}+\left|v_{k}^{S}(b)-v_{k}(b)\right|^{P}\right)}{2}} .
$$

In particular, for $P=1$, it can be expressed as follows:

$$
D_{k}=\frac{\left(\left|v_{k}^{S}(a)-v_{k}(a)\right|+\left|v_{k}^{S}(b)-v_{k}(b)\right|\right)}{2} .
$$

It is called the Hamming distance. For $P=2$, it can be expressed as follows:

$$
D_{k}=\sqrt{\frac{\left(\left|v_{k}^{S}(a)-v_{k}(a)\right|^{2}+\left|v_{k}^{S}(b)-v_{k}(b)\right|^{2}\right)}{2}} .
$$

It is called the Euclidean distance.

\section{Multiconstraint Fuzzy Prediction Analysis Algorithm and Model}

3.1. The Normalization of Multiple-Constraint Attributes. Different predictive analytics indicators may have different dimensions. In order to make the results of the predictive analysis more reliable, all predictive and analytical indicators need to have a unified measurement standard. Since it is a fuzzy prediction analysis, for the generality of the discussion, it is assumed that the attribute values of the prediction analysis are all fuzzy intervals, that is, the initial magnitude of the prediction analysis target with respect to the prediction analysis index $k$ is $v_{k}=\left[v_{k}(a), v_{k}(b)\right]$ and the normalized value is $u_{k}=\left[u_{k}(a), u_{k}(b)\right]$. The threshold value of the predictive analysis indicator $k$ is $v_{k}^{\Theta}=\left[v_{k}^{\Theta}(a), v_{k}^{\Theta}(b)\right]$, so the normalized formula of the cost-type predictive analysis indicator $k$ can be expressed as follows:

$$
\begin{gathered}
u_{k}=\left[u_{k}(a), u_{k}(b)\right], \\
u_{k}(a)=\frac{v_{k}^{\Theta}(b)-v_{k}(b)}{v_{k}^{\Theta}(b)-v_{k}^{\Theta}(a)}, \\
u_{k}(b)=\frac{v_{k}^{\Theta}(b)-v_{k}(a)}{v_{k}^{\Theta}(b)-v_{k}^{\Theta}(a)} .
\end{gathered}
$$

The normalized formula of benefit prediction and analysis indicators $k$ can be expressed as follows:

$$
\begin{gathered}
u_{k}=\left[u_{k}(a), u_{k}(b)\right], \\
u_{k}(a)==\frac{v_{k}(a)-v_{k}^{\Theta}(a)}{v_{k}^{\Theta}(b)-v_{k}^{\Theta}(a)}, \\
u_{k}(b)=\frac{v_{k}(b)-v_{k}^{\Theta}(a)}{v_{k}^{\Theta}(b)-v_{k}^{\Theta}(a)} .
\end{gathered}
$$

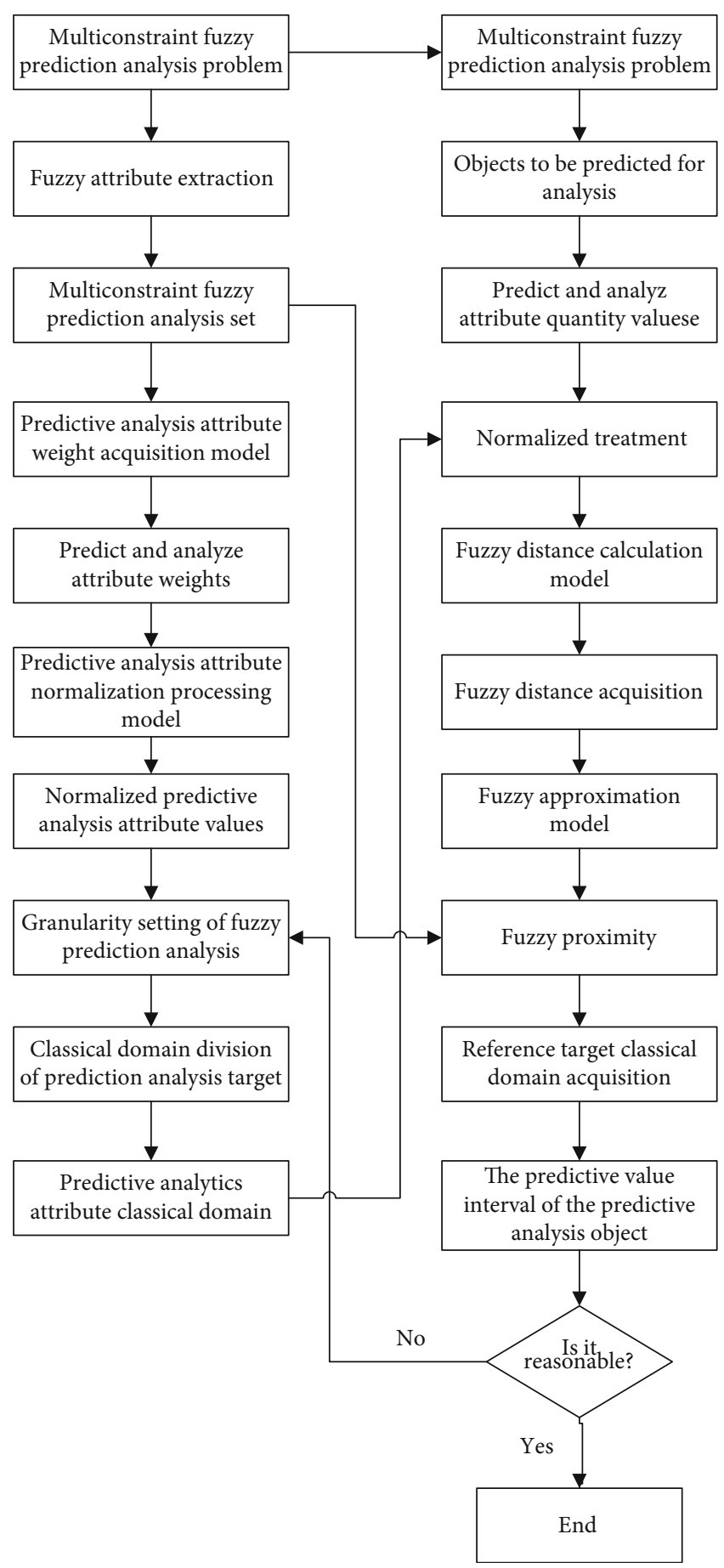

Figure 1: Implementation flow of the multiconstraint fuzzy prediction analysis.

3.2. Weight Handling of Multiple-Constraint Attributes. The multiconstraint attribute weights can be obtained by various methods, such as the AHP method, entropy weight method, comprehensive evaluation method, neural network method, and gray correlation method. AHP method has the advantages of simplicity, practicality, and reliable results. Therefore, this paper takes the AHP method as an example to illustrate the weight processing process of multiconstraint attributes. The process implementation is as follows [11]: 
TABle 1: The data comparison with reference [5].

\begin{tabular}{lcccr}
\hline & Classical field & Property 3 & Target range \\
\hline 1 & Property 1 & Property 2 & $0.120-0.180$ & $0.070-0.150$ \\
2 & $0-0.070$ & $0.135-0.195$ & $0.110-0.200$ & $0.150-0.230$ \\
3 & $0.070-0.180$ & $0.080-0.270$ & $0.340-0.400$ & $0.230-0.300$ \\
Prediction object & $0.130-0.340$ & $0.200-0.260$ & 0.309 & $/$ \\
Weight & 0.247 & 0.216 & 0.300 & \\
\hline
\end{tabular}

TABle 2: The result of the fuzzy distance comparison with reference [5].

\begin{tabular}{cccc}
\hline & & Classical field & \\
& Property 1 & Property 2 & Property 3 \\
\hline 1 & 0.215 & 0.059 & 0.162 \\
3 & 0.134 & 0.103 & 0.160 \\
\hline
\end{tabular}

(1) The first is forming a multiconstraint predictive analysis attribute set

(2) Expert judgement is carried out using a 1-9 ratio scale to obtain a judgment matrix $\mathbf{A}$

$$
\mathbf{A}=\left\{a_{i j}\right\}_{n x n}=\left[\begin{array}{cccc}
a_{11} & a_{12} & \cdots & \cdots \\
a_{21} & a_{22} & \cdots & \cdots \\
\cdots & \cdots & \cdots & \cdots \\
\cdots & \cdots & \cdots & a_{n n}
\end{array}\right]
$$

(3) Normalize the evaluation matrix $\mathbf{A}$

$$
\bar{a}_{j}=\sum_{i=1}^{n}\left(\frac{a_{i j}}{\sum_{i=1}^{n} a_{i j}}\right)
$$

(4) The maximum eigenvalue acquisition is performed on the evaluation matrix $\mathbf{A}$

$$
\lambda_{\max }=\sum_{j=1}^{n} \frac{(A W)_{i}}{n W_{i}}
$$

(5) Perform a consistency check on the evaluation matrix A

$$
\begin{aligned}
C I & =\frac{\lambda_{\max }-n}{n-1}, \\
C R & =\frac{C I}{R I} .
\end{aligned}
$$

If $C R \leq 0.1$, it indicates that the evaluation matrix $\mathbf{A}$ has good consistency and the next step can be per-
TABLE 3: The result of the fuzzy approximation comparison with reference [5].

\begin{tabular}{ccccc}
\hline & Property 1 & $\begin{array}{c}\text { Classical field } \\
\text { Property 2 }\end{array}$ & Property 3 & $\begin{array}{c}\text { Weighted fuzzy } \\
\text { approximation }\end{array}$ \\
\hline 1 & 0.785 & 0.942 & 0.838 & 0.864 \\
2 & 0.866 & 0.897 & 0.840 & 0.870 \\
3 & 0.894 & 0.967 & 0.932 & 0.937 \\
\hline
\end{tabular}

formed. Otherwise, the evaluation matrix needs to be reset

(6) Weight acquisition

$$
w_{j}=\frac{\bar{a}_{j}}{\sum_{j=1}^{n} \bar{a}_{j}}
$$

3.3. Multiconstraint Attribute Predictive Analysis Granularity Setting. The granularity setting of multiconstraint attribute predictive analysis is to set the interval division of the quantitative value of the multiconstraint fuzzy predictive analysis attribute set. The granularity should be selected appropriately, neither too large nor too small, and should be analyzed and selected according to the actual situation of the prediction analysis target. If the selected granularity is too small, the attribute set of multiconstraint fuzzy prediction analysis is divided too fine. In extreme cases, each data item is a reference target, which may lead to the failure to predict the target object. If the selected granularity is too large, the division of the multiconstraint fuzzy prediction analysis attribute set is too thick, which will lead to a too wide range of reference target and too wide range of prediction results, so that the target object cannot be accurately predicted.

3.4. Classical Domain Generation of Multiconstraint Properties. On the basis of setting the granularity of multiconstraint attribute prediction analysis, assuming that it is divided into $H$ classical domains, the quantitative value interval of the reference target of the corresponding classical domain on the attribute set of fuzzy prediction analysis can be obtained $Z_{h}=\left[Z_{h}(a), Z_{h}(b)\right]$.

According to the magnitude of the interval $Z_{h}=\left[Z_{h}(a)\right.$, $\left.Z_{h}(b)\right]$, the reference objects corresponding to the historical 
TABLE 4: Comparison and analysis data with literature [11].

\begin{tabular}{lccccc}
\hline & & \multicolumn{2}{c}{ Classical field } & \multicolumn{2}{c}{ Target range } \\
\hline 1 & Property 1 & Property 2 & Property 3 & Property 4 & $0.080-0.180$ \\
2 & $0.069-0.158$ & $0.085-0.327$ & $0.146-0.196$ & 0.162 & $0.180-0.280$ \\
3 & $0.083-0.181$ & $0.172-0.265$ & $0.127-0.275$ & $0.106-0.216$ & $0.280-0.400$ \\
Object to be predicted & $0.187-0.327$ & $0.256-0.284$ & $0.292-0.332$ & $0.209-0.301$ & 0.183 \\
The weight & 0.195 & 0.202 & 0.227 & 0.081 & $/$ \\
\hline
\end{tabular}

records contained therein are determined. Assuming that the number of reference objects is $N$, the classical field $\Omega_{k}^{h}$ of the reference target $h$ formed for these reference objects is

$$
\begin{aligned}
\Omega_{k}^{h} & =\left[\Omega_{k}^{h}(a), \Omega_{k}^{h}(b)\right], \\
\Omega_{k}^{h}(a) & \leq \min _{1 \leq i \leq N}\left(u_{k}=\left[u_{k}^{i}(a), u_{k}^{i}(b)\right]\right), \\
\Omega_{k}^{h}(b) & \geq \max _{1 \leq i \leq N}\left(u_{k}=\left[u_{k}^{i}(a), u_{k}^{i}(b)\right]\right) .
\end{aligned}
$$

In general, in order to ensure the reliability and accuracy of the prediction results, take the following values, even if the range is a little bit wider.

$$
\begin{aligned}
& \Omega_{k}^{h}(a)<\min _{1 \leq i \leq N} u_{k}^{i}(a), \\
& \Omega_{k}^{h}(b)>\max _{1 \leq i \leq N} u_{k}^{i}(b) .
\end{aligned}
$$

3.5. Multiconstraint Attribute Fuzzy Proximity Calculation. Assume that prediction target on the forecasting analysis index $k$ value for $u_{k}=\left[u_{k}(a), u_{k}(b)\right]$, according to the fuzzy distance calculation formula given above, the fuzzy distance $D_{k}^{h}$ between the prediction and analysis target $k$, and the reference target $h$ can be obtained.

$$
D_{k}^{h}=\sqrt[P]{\frac{\left(\left|\Omega_{k}^{h}(a)-u_{k}(a)\right|^{P}+\left|\Omega_{k}^{h}(b)-u_{k}(b)\right|^{P}\right)}{2}} .
$$

When the magnitude of the predictive analysis target with respect to the predictive analysis index $k$ is an exact value, that is, $u_{k}=u_{k}(a)=u_{k}(b)$, it is described as follows:

$$
D_{k}^{h}=\sqrt[P]{\frac{\left(\left|\Omega_{k}^{h}(a)-u_{k}\right|^{P}+\left|\Omega_{k}^{h}(b)-u_{k}\right|^{P}\right)}{2}} .
$$

The greater the fuzzy distance $D_{k}^{h}$, the more distant the two are, and the smaller the fuzzy distance $D_{k}^{h}$ is, the closer they are. Therefore, the fuzzy proximity $\Phi_{k}^{h}$ is introduced

$$
\Phi_{k}^{h}=1-D_{k}^{h}
$$

Assuming that the weight of each predictive analysis attribute is taken into account, the comprehensive weighted fuzzy approximation $\Phi_{k}^{h}$ is

$$
\Phi^{h}=\sum_{k=1}^{K}\left(w_{k} *\left(1-D_{k}^{h}\right)\right)
$$

3.6. Implementation of Multiconstrained Fuzzy Prediction Analysis. The larger the integrated weighted fuzzy proximity $\Phi_{k}^{h}$ is, the closer the predicted analysis target is to the reference object, and vice versa, the farther away the predicted analysis target is from the reference object. Therefore, based on the comprehensive weighted fuzzy proximity $\Phi_{k}^{h}$, the predicted analysis target can be obtained according to the size of the integrated weighted fuzzy proximity $\Phi_{k}^{h}$ and the corresponding domain value range $Z_{h}=\left[Z_{h}(a), Z_{h}(b)\right]$ of the corresponding reference target $h$. Then, the fuzzy predictive value $Z^{*}$ of the prediction analysis target must satisfy the following equation.

$$
Z^{*} \in Z_{h}=\left[Z_{h}(a), Z_{h}(b)\right]
$$

Thus, the realization process of the multiconstraint fuzzy prediction analysis algorithm can be shown in Figure 1.

\section{Comparative Analysis}

In order to verify the effectiveness and feasibility of the proposed multiconstraint fuzzy prediction analysis algorithm, the Euclidean distance calculation formula is used for analysis. At the same time, the implementation process of the algorithm and model proposed in this paper is explained.

Compared with the data in literature [5], the initial comparison data is shown in Table 1.

According to the calculation formulas of the fuzzy distance and fuzzy proximity, the corresponding calculation results can be obtained, as shown in Tables 2 and 3, respectively.

It can be seen that the predicted result target interval of this algorithm should be 0.937 . This is consistent with the results of literature [5]. However, it should be pointed out that the predicted object is closer to the target interval 1 for attribute 1 and closer to the target interval 2 for attribute 2 . For attribute 3 , the difference is not obvious, but because attribute 2 accounts for a large proportion, the difference should not be obvious, which is consistent with the calculation results. 
TABle 5: Comparison of the fuzzy distance calculation results with literature [11].

\begin{tabular}{ccccc}
\hline & \multicolumn{4}{c}{ Classical field } \\
& Property 1 & Property 2 & Property 3 & Property 4 \\
\hline 1 & 0.093 & 0.121 & 0.061 & 0.076 \\
2 & 0.080 & 0.049 & 0.078 & 0.059 \\
3 & 0.094 & 0.069 & 0.087 & 0.085 \\
\hline
\end{tabular}

TABle 6: Calculation results of fuzzy proximity compared with literature [11].

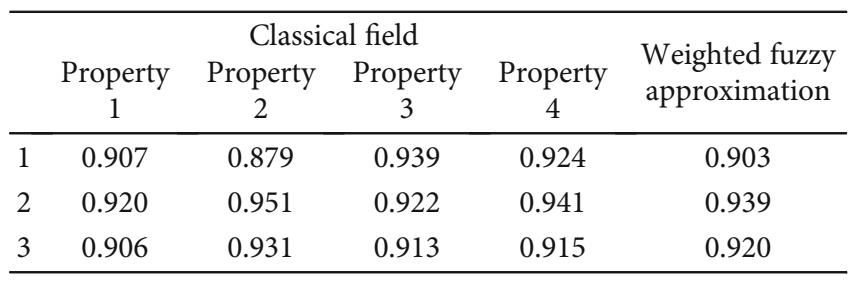

Similarly, the same processing method was used for comparative analysis with literature [11] and the calculation results were shown in Tables 4, 5, and 6.

According to the fuzzy distance and fuzzy proximity calculation formula in the paper, corresponding calculation results can be obtained, as shown in Tables 2 and 3, respectively.

According to the data in the table, the target interval of the prediction results of this algorithm should be 0.939 corresponding to the target interval, that is, $0.180-0.280$, which is consistent with the result of reference [11].

\section{Conclusion}

Due to the existence of fuzzy information, the prediction and analysis of complex systems under multiple constraints become very complex. Therefore, the study of multiconstraint fuzzy prediction analysis method has important engineering value. An improved multiconstraint fuzzy prediction analysis algorithm is proposed. The algorithm focuses on the establishment of the attribute constraint set, standardization, weight and prediction analysis of the granularity set, generation of the classical domain, calculation of fuzzy distance and fuzzy closeness, constraint fuzzy prediction analysis, and implementation of the algorithm and model. At the same time, in order to verify the effectiveness and feasibility of the proposed algorithm, this paper compares and analyzes the proposed algorithm and related algorithms. The results are consistent, which provides a new solution and method support for multiconstraint fuzzy prediction analysis.

\section{Data Availability}

The labeled dataset used to support the findings of this study is available from the corresponding author upon request.

\section{Conflicts of Interest}

The author declares no competing interests.

\section{Acknowledgments}

TangSeng Huang helped perform the analysis with constructive discussions. This study is supported by the Sichuan Provincial Department of Education project (18ZA0419), the educational reform project of Sichuan University of Arts and Science (2020JY014), the and first-class course project of Sichuan University of Arts and Science (2020KCC026).

\section{References}

[1] L. Zhang, W. Tang, Y. Liu, and T. Lv, "Multiobjective optimization and decision-making for DG planning considering benefits between distribution company and DGs owner," International Journal of Electrical Power \& Energy Systems, vol. 73, pp. 465-474, 2015.

[2] T. Dun, Y. Jun, and D. Min, "Object-oriented multiple poles and multi-objective constraint optimization design method," Machine Building \& Automation, vol. 4, pp. 1-4, 2016.

[3] L. Peng, H. Min, and H. Ke, "Research on the human resource allocation of scientific research projects based on multiobjective optimization," Computer Applications and Software, vol. 34, no. 5, pp. 217-222, 2017.

[4] Z. Su, "Prediction of demands on cultivated land based on multi-criteria decision," Hubei Agricultural Sciences, vol. 53, no. 17, pp. 4219-4243, 2014.

[5] L. Ya, L. Jun, L. Jin, and X. Xiao, "Research and application of mobile phone gray fault rate based on gray model," Computer Applications and Software, vol. 35, no. 2, pp. 112-116, 2018.

[6] Z. Zhi, T. Nan, and Y. Hong, "On analysis and prediction of schooling population in compulsory education of Tianjin based on multi-factor gray model and recursive calculation method," Journal of Southwest China Normal University (Natural Science Edition), vol. 42, no. 3, pp. 49-55, 2017.

[7] L. Wei, G. Xiao, and M. Yin, "Research and application of uncertain genetic neural network in landslide hazard prediction," Computer Engineering, vol. 43, no. 2, pp. 308-316, 2017.

[8] X. Ting, W. Wen, and D. Liang, "Construction land demand forecasting of Chengdu city based on PCA-RBF neural network," Journal of Southwest University (Natural Science), vol. 39, no. 11, pp. 183-190, 2014.

[9] Z. Pan, X. Shou, and L. Jun, "A prediction model of water content of fresh tobacco leaves based on support vector machine regression," Journal of Southwest University (Natural Science), vol. 38, no. 4, pp. 165-170, 2016.

[10] Y. Jing, B. Qiang, L. Jie, and W. Fu, "Construction of aggregation quality predicting model for digital resource in library based on improved genetic algorithm and BP neural network," Data Analysis and Knowledge Discovery, vol. 1, no. 12, pp. 4962, 2017.

[11] M. Zhou, Y. Long, W. Zhang et al., "Adaptive genetic algorithm-aided neural network with channel state information tensor decomposition for indoor localization," IEEE Transactions on Evolutionary Computation, p. 1, 2021.

[12] Q. Guo and G. Zou, "Prediction methods for extension architecture programming based on decision tree classification," CAAI Transactions on Intelligent Systems, vol. 12, no. 1, pp. 117-123, 2017.

[13] S. Li and A. X. Ou, “The effectiveness students' ideological evaluation model of university and political education based 
on the fuzzy mathematics theory," Mathematics in Practice and Theory, vol. 45, no. 13, pp. 299-306, 2015.

[14] M. M. Silva, A. P. de Gusmão, T. Poleto, L. C. Silva, and A. P. Costa, "A multidimensional approach to information security risk management using FMEA and fuzzy theory," International Journal of Information Management, vol. 34, no. 6, pp. 733-740, 2014.

[15] M. Zhou, X. Li, Y. Wang, S. Li, Y. Ding, and W. Nie, “6G multisource information fusion based indoor positioning via Gaussian kernel density estimation," IEEE Internet of Things Journal, p. 1,2020 .

[16] M. Zhou, Y. Wang, Y. Liu, and Z. Tian, "An informationtheoretic view of WLAN localization error bound in GPSdenied environment," IEEE Transactions on Vehicular Technology, vol. 68, no. 4, pp. 4089-4093, 2019.

[17] M. Zhou, Y. Wang, Z. Tian, Y. Lian, Y. Wang, and B. Wang, "Calibrated data simplification for energy-efficient location sensing in internet of things," IEEE Internet of Things Journal, vol. 6, no. 4, pp. 6125-6133, 2019.

[18] S. K. Shiroodi, M. Ghafoori, H. R. Ansari, G. Lashkaripour, and M. Ghanadian, "Shear wave prediction using committee fuzzy model constrained by lithofacies, Zagros basin, SW Iran," Journal of African Earth Sciences, vol. 126, pp. 123135, 2017.

[19] Y. Yan and B. Su, "Design of explicit fuzzy prediction controller for constrained nonlinear systems," Mathematical Problems in Engineering, vol. 2015, Article ID 857546, 7 pages, 2015. 